\title{
Correction
}

\section{Correction: Suzuki et al., "Cadherin-8 Is Required for the First Relay Synapses to Receive Functional Inputs} from Primary Sensory Afferents for Cold Sensation"

In the article "Cadherin-8 Is Required for the First Relay Synapses to Receive Functional Inputs from Primary Sensory Afferents for Cold Sensation” by Sachihiro C. Suzuki, Hidemasa Furue, Kohei Koga, Nan Jiang, Mitsuo Nohmi, Yuka Shimazaki, Yuko KatohFukui, Minesuke Yokoyama, Megumu Yoshimura, and Masatoshi Takeichi, which appeared on pages 3466-3476 of the March 28, 2007 issue, the authors regret an error in the mix-up of some blots listed in Supplemental Figure 3. This mistake only affects the supplemental material and does not affect the main discovery and conclusion of the paper. A corrected Supplemental Figure 3 is provided below.

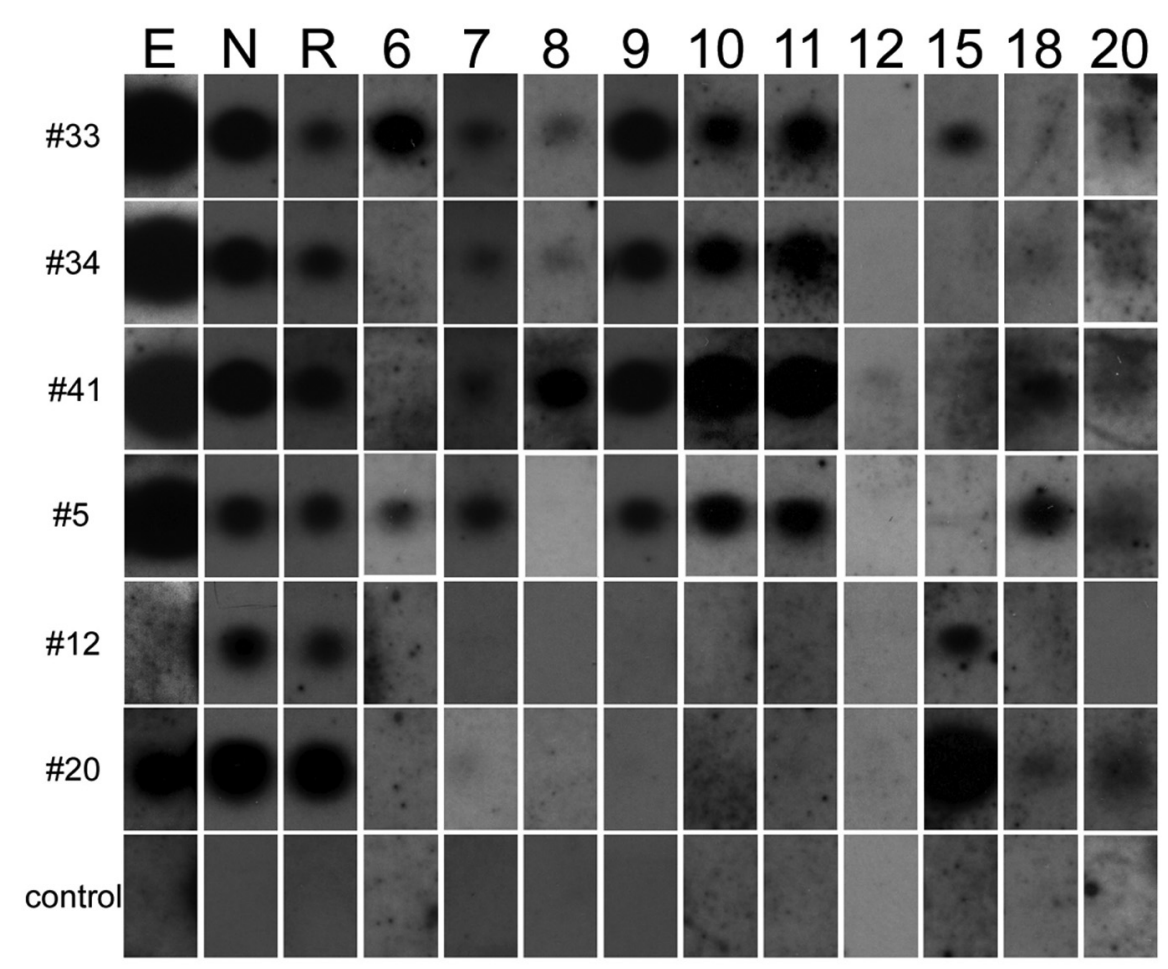

Figure 3. 